\title{
Virtual reality simulator for vitreoretinal surgery using integrated OCT data
}

This article was published in the following Dove Press journal:

Clinical Ophthalmology

3I March 2014

Number of times this article has been viewed

\author{
Igor Kozak' \\ Pat Banerjee ${ }^{2}$ \\ Jia Luo ${ }^{2}$ \\ Cristian Luciano ${ }^{2}$ \\ 'King Khaled Eye Specialist Hospital, \\ Vitreoretinal Division, Riyadh, \\ Kingdom of Saudi Arabia; ${ }^{2}$ College \\ of Engineering, University of Illinois \\ at Chicago, Chicago, IL, USA
}

Correspondence: Igor Kozak King Khaled Eye Specialist Hospital, PO Box 7I9I, Riyadh, Kingdom of Saudi Arabia Tel +966 |482 | 234 ext 3772 Fax+966 I482 I234 ext 3727

Email ikozak@kkesh.med.sa

\begin{abstract}
Operative practice using surgical simulators has become a part of training in many surgical specialties, including ophthalmology. We introduce a virtual reality retina surgery simulator capable of integrating optical coherence tomography (OCT) scans from real patients for practicing vitreoretinal surgery using different pathologic scenarios.
\end{abstract}

Keywords: optical coherence tomography

\section{Introduction}

Surgical simulation has been used in several surgical specialties to provide assessment and acquisition of skills for particular surgical procedures. ${ }^{1}$ Traditional wet laboratory or animal environments have become either obsolete or ethically unacceptable. Ophthalmic surgery simulators are a popular training tool for basic surgical skills acquisition before entering clinical practice.

Currently available simulators for ocular surgery include EYESi (VRmagic, Mannheim, Germany), PhacoVision (Melerit Medical, Linköping, Sweden), and MicroVisTouch (Immersive Touch Inc., Chicago, IL, USA). All three simulators use the stereoscopic microscope while practicing the steps of cataract surgery. The first simulator has been in use the longest, and therefore, construct validity for this device has been demonstrated for many steps of cataract surgery. ${ }^{2-4}$ The Micro VisTouch surgical simulator has been used and validated predominantly in applications other than ophthalmology. 5,6 Therefore, the number of published articles in ophthalmic literature for both devices is limited. At this time, EYESi simulator is the only device that has been validated in peer-reviewed publications and is available on the market for the training of both cataract and vitreoretinal surgery. ${ }^{2-4,7}$

Among the available simulators, the MicroVisTouch simulator is the only device that has incorporated a haptic (tactile) feedback interface. As per published surveys, the integration of the tactile feedback has been shown to provide more realistic operative experience among ophthalmic surgeons. ${ }^{8}$ Furthermore, the MicroVisTouch simulator is the only device that provides a full virtual experience, which includes the instruments and the head and eye of the virtual patient. The trainee works with virtual tissues in the space. In contrast, both PhacoVision and EYESi devices use an immobile physical head and eye.

In an attempt to follow the fast-evolving advances in retinal surgery, we feel there is an unmet need for advanced surgical simulator training in vitreoretinal interface diseases. The integration of retinal images from the 3D optical coherence tomography 
(OCT) volume scans, which could serve as a disease template, is a further step to bring surgical simulation closer to reality. This report for the first time describes the integration of OCT images into a virtual reality surgical simulator.

\section{Methods}

For the purpose of the study we used random, previously acquired patients' images from the database of the King Khaled Eye Specialist Hospital in Riyadh, Saudi Arabia. Eyes with epiretinal membrane $(n=3)$ and macular hole $(n=2)$ were imaged using the RTVue instrument (Optovue, Freemont, CA, USA). The image raw data were downloaded, exported with no patient identifier, and used for 3D rendering in MicroVisTouch. The study adhered to the tenets of the Declaration of Helsinki in clinical research.

We then designed a computer-based simulator for epiretinal membrane (ERM) and internal limiting membrane (ILM) peeling procedures with the idea to develop a high performance haptics-, physics-, and graphics-enabled simulator. The study was conducted on the MicroVisTouch surgical simulator (Figure 1), which has the following specifications: Dell

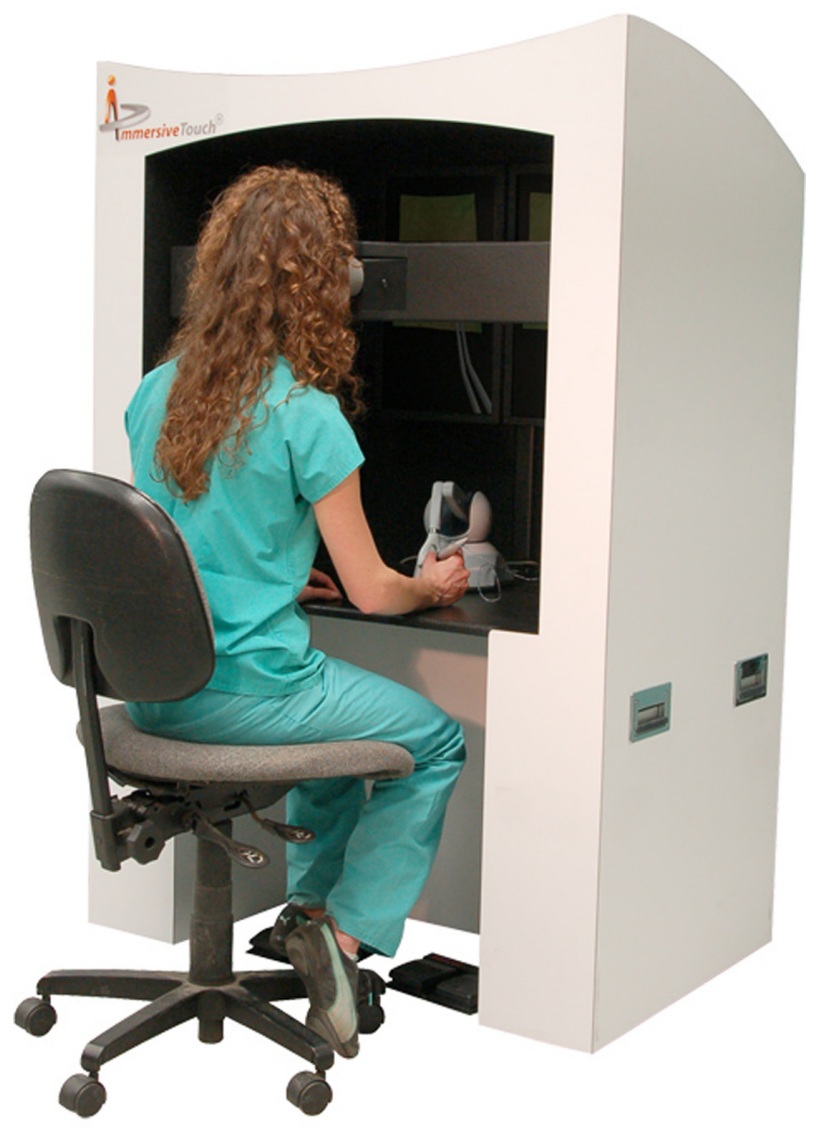

Figure I An image of an operator using virtual reality system MicroVisTouch (ImmersiveTouch, Chicago, IL, USA) using haptic (tactile) feedback and stereo viewer for vitreoretinal surgery simulation. precision T7500 with Intel Xeon X5690 6 cores CPU, 12GB RAM, NVIDIA Quadro FX 6000 graphics card, SensAble Phantom Omni haptic device, and Microsoft Windows 7 64-bit OS. High fidelity haptic feedback is rendered to provide the sense of real surgery sensations. Opening and closing of the forceps is governed by a pressure sensor attached to the Geomagic Touch haptic device. The deformable retina is modeled from volumetric data generated from the OCT scan (Optovue) using position-based dynamics with improved performance over traditional mass-spring models.

\section{Results}

A customized peeling algorithm with full controllability was developed to simulate the peeling of the ERM and ILM. Figure 2 shows an example of a successful integration of an OCT image from a real patient with vitreoretinal interface abnormality which will serve as a template for ERM removal practice. The surface membranes can be distinguished from retinal tissue on the integrated OCT scan. A combination of vertex and fragment shaders is adopted to provide improved realism on graphical/visual effect. The forceps are used for surgical simulation and the user is instructed to apply enough pressure to the sensor in order for the membrane not to slip out of the forceps.

Several metrics can be used to assess surgical performance. The circularity score measures how perfect the circle of removed ERM or ILM is with respect to an ideal peel. The accuracy score indicates the number of times the retina is touched by the forceps.

The fluency score indicates the number of times the forceps are closed to grab the membrane. These all can be used as part of surgical skills evaluation both at initial performance and subsequent sessions.

\section{Discussion}

Virtual reality is a computer-simulated environment that simulates physical presence in places and where the user interacts with graphic software which can provide visual, tactile, and auditory feedback. Ophthalmic surgical simulation is becoming increasingly popular, and so far, is most commonly used for cataract surgery practice. Recently, the International Forum of Ophthalmic Simulation has been created to enhance training programs and educational curriculum in surgical simulations. ${ }^{9,10}$

While cataract surgery is routinely trained on simulators, retinal surgical simulation is in general less common. Vitreoretinal surgery is a complex surgery requiring a high level of dexterity and coordination, and the learning 

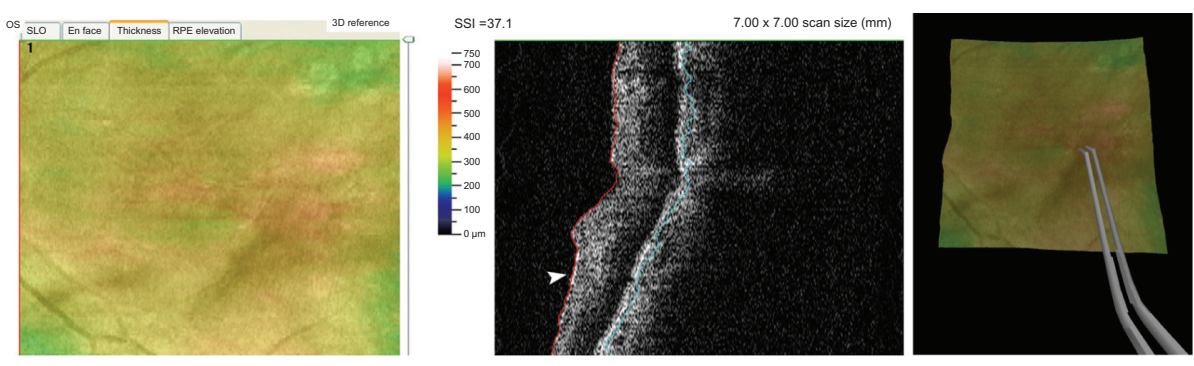

Figure 2 Left panel: posterior pole image of an eye with epiretinal membrane and surface wrinkling. Central panel: OCT B-scan of the same eye showing retinal wrinkling and an epiretinal membrane (arrowhead). Right panel: the same OCT image integrated into a surgical simulator with an operator practicing epiretinal membrane removal using virtual forceps.

Abbreviation: OCT, optical coherence tomography.

curve can be much longer compared to other ophthalmic procedures. Few vitreoretinal surgical simulators have been introduced in the past and their use has remained limited. Hikichi et al proposed a virtual technology system consisting of four major parts: high-resolution color stereo binoculars, haptic devices, foot switches, and a high-speed graphics computer. The system was designed for the practice of removing pre- and sub-retinal membranes. ${ }^{11}$ Wagner et al showed a computer-based medical training workstation for the simulation of intraocular eye surgery in which the surgeon manipulates two instruments inside a mechanical model of the eye. The instrument positions are tracked by charge-coupled device (CCD) cameras and monitored by a PC which renders the scenery using a computer-graphic model of the eye and the instruments. ${ }^{12}$ Later, Verma et al presented another virtual reality vitreoretinal simulator simulating the initial steps of vitreoretinal surgery such as vitreous removal. Soft tissue computer modeling was carried out to mimic the removal of vitreous opacities. ${ }^{13}$ At present, the most common device for practicing steps in retina surgery is the EyeSi system, which uses a manikin head. The surgical instruments are visualized within the model eye via sensors that are used to gauge instrument position and movement. No tactile feedback with ocular tissues is provided by the simulator. The user can select contact lens or binocular indirect opthalmomicroscope (BIOM) visualization as well as a variety of virtual surgical instruments such as a light pipe, forceps, scissors, and vitrectomy hand pieces. The EyeSi ${ }^{\circledR}$ records performance metrics that include iatrogenic injuries. It has been used in a curriculum to enhance ophthalmic surgical training. ${ }^{14}$

A new concept in surgical simulation is haptic technology with manipulation in virtual space rather than touching a physical organ model. The PHANTOM ${ }^{\circledR}$ Omni ${ }^{\mathrm{TM}}$ model by SensAble Phantom Omni (Woburn, MA, USA) is a widely used haptic device enabling 3D touch-enabled systems for medical modeling. It has been used in several specialties. ${ }^{15-17}$ Similarly, MicroVisTouch uses a virtual reality haptic feedback approach for simulation and has been validated in surgical specialties. ${ }^{6}$ It has also been applied to ophthalmology in cataract surgery simulation. Banerjee et al investigated the concurrent validity of capsulorhexis performance metrics (duration, number of capsular grabs per completed capsulorhexis, and circularity of the capsulorhexis) and compared it to live surgeries. ${ }^{18}$

In this report, we are interested in using the concept of virtual reality with haptic technology for vitreoretinal surgery and in customizing the training to existing disease from real patients rather than repeatedly presenting the same model and task to a trainee. For this reason, we integrated OCT scans of vitreoretinal conditions to a surgical simulator. The integrated images serve as an image template for practicing surgical steps. Similar to cataract surgery simulation with this technology, several metrics can be used to assess surgical performance. The circularity score measures how perfect the circle of removed ERM or ILM is with respect to an ideal peel. The accuracy score indicates the number of times the retina is touched by the forceps. The fluency score indicates the number of times the forceps are closed to grab the membrane. These can all be used as part of surgical skills evaluation both at initial performance and subsequent sessions. Further studies are needed to validate this approach in comparison to live surgeries.

In summary, the concept introduced here can be utilized to practice image-based vitreoretinal surgery using OCT scans from real patients. Contrary to other surgical simulators which use a standardized pre-operative starting condition, the integration of retinal imaging into a surgical simulator will allow students to be challenged with numerous different conditions. We believe this innovation will contribute to enhancing preclinical surgical experience in vitreoretinal surgery. 


\section{Disclosure}

$\mathrm{PB}, \mathrm{JL}$, and $\mathrm{CL}$ have recevied research support from Immersive Touch, Inc. (Chicago, IL, USA). The authors report no other conflicts of interest in this work.

\section{References}

1. Cook DA, Hatala R, Brydges R, et al. Technology-enhanced simulation for health professions education: a systematic review and meta-analysis. JAMA. 2011;306(9):978-988.

2. Mahr MA, Hodge DO. Construct validity of anterior segment anti-tremor and forceps surgical simulator training modules: attending versus resident surgeon performance. J Cataract Refract Surg. 2008;34(6):980-985.

3. Feudner EM, Engel C, Neuhann IM, et al. Virtual reality training improves wet-lab performance of capsulorhexis: results of a randomized, controlled study. Graefes Arch Clin Exp Ophthalmol. 2009;247(7):955-963.

4. Privett B, Greenlee E, Rogers G, Oetting TA. Construct validity of a surgical simulator as a valid model for capsulorrhexis training. J Cataract Refract Surg. 2010;36(11):1835-1838.

5. Alaraj A, Lemole MG, Finkle JH, et al. Virtual reality training in neurosurgery: review of current status and future applications. Surg Neurol Int. 2011;2:52.

6. Roitberg B, Banerjee P, Luciano C, et al. Sensory and motor skill testing in neurosurgery applicants: a pilot study using a virtual reality haptic neurosurgical simulator. Neurosurgery. 2013;Suppl 1:S116-S121.

7. Rossi JV, Verma D, Fujii GY, et al. Virtual vitreoretinal surgical simulator as a training tool. Retina. 2004;24:231-236.
8. Huynh N, Akbari M, Loewenstein JI. Tactile feedback in cataract and retinal surgery: a survey-based study. $J$ Acad Ophthalmol. 2008;1: $79-85$.

9. Saleh GM, Lamparter J, Sullivan PM, et al. The international forum of ophthalmic simulation: developing a virtual reality training curriculum for ophthalmology. Br J Ophthalmol. 2013;97(6):789-792.

10. Gillan SN, Saleh GM. Ophthalmic surgical simulation: a new era. JAMA Ophthalmol. 2013;131(12):1623-1624.

11. Hikichi T, Yoshida A, Igarashi S, et al. Vitreous surgery simulator. Arch Ophthalmol. 2000;118:1679-1681.

12. Wagner C, Schill M, Hennen M, et al. [Virtual reality in ophthalmological education]. Ophthalmologe. 2001;98(4):409-413. German.

13. Verma D, Wills D, Verma M. Virtual reality simulator for vitreoretinal surgery. Eye. 2003;17:71-73.

14. Grodin MH, Johnson MT, Acree JL, Glaser BM. Ophthalmic surgical training: a curriculum to enhance surgical simulation. Retina. 2008;28(10):1509-1514.

15. Sorensen MS, Mosegaard J, Trier P. The visible ear simulator: a public PC application for GPU-accelerated haptic 3D simulation of ear surgery based on the visible ear data. Otol Neurotol. 2009;30(4):484-487.

16. Lerman IR, Souzdalnitsky D, Narouze S. A low-cost, durable, combined ultrasound and fluoroscopic phantom for cervical transforaminal injections. Reg Anesth Pain Med. 2012;37(3):344-348.

17. Khalifa YM, Bogorad D, Gibson V, Peifer J, Nussbaum J. Virtual reality in ophthalmology training. Surv Ophthalmol. 2006;51(3):259-273.

18. Banerjee PP, Edward DP, Liang S, et al. Concurrent and face validity of a capsulorhexis simulation with respect to human patients. Stud Health Technol Inform. 2012;173:35-41.
Clinical Ophthalmology

\section{Publish your work in this journal}

Clinical Ophthalmology is an international, peer-reviewed journal covering all subspecialties within ophthalmology. Key topics include: Optometry; Visual science; Pharmacology and drug therapy in eye diseases; Basic Sciences; Primary and Secondary eye care; Patient Safety and Quality of Care Improvements. This journal is indexed on Submit your manuscript here: http://www.dovepress.com/clinical-ophthalmology-journal

\section{Dovepress}

PubMed Central and CAS, and is the official journal of The Society of Clinical Ophthalmology (SCO). The manuscript management system is completely online and includes a very quick and fair peer-review system, which is all easy to use. Visit http://www.dovepress.com/ testimonials.php to read real quotes from published authors. 